\title{
Mathematical method to build an empirical model for inhaled anesthetic agent wash-in
}

\author{
Jan FA Hendrickx ${ }^{1 *}$, Harry Lemmens ${ }^{2}$, Sofie De Cooman ${ }^{3}$, André AJ Van Zundert ${ }^{4}$, René EJ Grouls ${ }^{5}$, Eric Mortier ${ }^{6}$ \\ and Andre M De Wolf ${ }^{7}$
}

\begin{abstract}
Background: The wide range of fresh gas flow - vaporizer setting (FGF - $F_{D}$ ) combinations used by different anesthesiologists during the wash-in period of inhaled anesthetics indicates that the selection of FGF and $F_{D}$ is based on habit and personal experience. An empirical model could rationalize FGF - $F_{D}$ selection during wash-in.

Methods: During model derivation, 50 ASA PS I-Il patients received desflurane in $\mathrm{O}_{2}$ with an $\mathrm{ADU}^{\circledR}$ anesthesia machine with a random combination of a fixed FGF - $F_{D}$ setting. The resulting course of the end-expired desflurane concentration $\left(F_{A}\right)$ was modeled with Excel Solver, with patient age, height, and weight as covariates; NONMEM was used to check for parsimony. The resulting equation was solved for $F_{D}$, and prospectively tested by having the formula calculate $F_{D}$ to be used by the anesthesiologist after randomly selecting a $F G F$, a target $F_{A}\left(F_{A t}\right)$, and a specified time interval $(1-5 \mathrm{~min})$ after turning on the vaporizer after which $\mathrm{F}_{\mathrm{At}}$ had to be reached. The following targets were tested: desflurane $F_{\text {At }} 3.5 \%$ after $3.5 \min (n=40), 5 \%$ after $5 \min (n=37)$, and $6 \%$ after 4.5 $\min (n=37)$.

Results: Solving the equation derived during model development for $F_{D}$ yields $F_{D}=-\left(e^{\left(-F G F^{*}-0.23+F G F^{*} 0.24\right) *}\left(e^{\left(F G F^{*}-0.23\right)}\right.\right.$

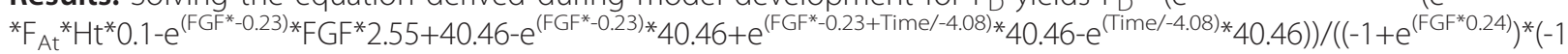
$\left.\left.+\mathrm{e}^{(\mathrm{Time} /-4.08)}\right)^{*} 39.29\right)$. Only height $(\mathrm{Ht})$ could be withheld as a significant covariate. Median performance error and median absolute performance error were -2.9 and $7.0 \%$ in the $3.5 \%$ after 3.5 min group, -3.4 and $11.4 \%$ in the $5 \%$ after 5 min group, and -16.2 and $16.2 \%$ in the $6 \%$ after 4.5 min groups, respectively.

Conclusions: An empirical model can be used to predict the FGF - $F_{D}$ combinations that attain a target endexpired anesthetic agent concentration with clinically acceptable accuracy within the first 5 min of the start of administration. The sequences are easily calculated in an Excel file and simple to use (one fixed FGF - FD setting), and will minimize agent consumption and reduce pollution by allowing to determine the lowest possible FGF that can be used. Different anesthesia machines will likely have different equations for different agents.
\end{abstract}

\section{Background}

What fresh gas flow - vaporizer setting $\left(\mathrm{FGF}-\mathrm{F}_{\mathrm{D}}\right)$ combination should be used for a particular patient when starting the administration of potent inhaled anesthetics to reach a target end-expired concentration $\left(\mathrm{F}_{\mathrm{A}}\right)$ after a predetermined time interval without excessively wasting potent inhaled anesthetic? The wide range of FGF - $F_{D}$ combinations used by different anesthesiologists during the wash-in period of potent inhaled anesthetics indicates that the selection of FGF and $F_{D}$ is based on habit and

\footnotetext{
*Correspondence: jenwahendrickx@yahoo.com

'Department of Anesthesiology, Intensive Care and Pain Therapy, Onze Lieve Vrouwziekenhuis, Aalst, Belgium

Full list of author information is available at the end of the article
}

personal experience. Some anesthesiologists use a high FGF (to shorten the wash-in time constant of the anesthesia circle breathing system, and to avoid rebreathing that results in dilution of $F_{D}$ ), while others prefer to use a lower FGF in combination with a higher $\mathrm{F}_{\mathrm{D}}$ (to compensate for the longer wash-in time constant, and to reduce agent consumption). While all anesthesiologists swiftly attain the target $\mathrm{F}_{\mathrm{A}}\left(\mathrm{F}_{\mathrm{At}}\right)$ because $\mathrm{FGF}$ and $\mathrm{F}_{\mathrm{D}}$ can be adjusted according to the measured $\mathrm{F}_{\mathrm{A}}$, it is unlikely that the particular FGF - $\mathrm{F}_{\mathrm{D}}$ combination used was that with the least number of $F_{D}$ and FGF adjustments and minimum waste. The use of high FGF, even for a seemingly brief period ( $5 \mathrm{~min})$, may increase agent consumption above that of an ensuing one hour maintenance phase

\section{Ciomed Central}


with a $1 \mathrm{~L}$. $\min ^{-1}$ FGF [1], and may forfeit the savings of an automated closed-circuit anesthesia machine [2].

Instead of relying on personal preference, we hypothesize that very specific FGF - $F_{D}$ combinations can be used in the individual patient to attain a $F_{\text {At }}$ within a specified time interval by using an empirical model of the kinetics of inhaled anesthetics during wash-in. Simple, easy to remember FGF - $F_{D}$ combinations construed from these models could reduce agent consumption while not distracting the anesthesiologist from other tasks during the induction period of anesthesia [3].

While kinetics of inhaled anesthetics in the anesthesia circle system have already been modeled using mass balances [4-6], few of these models have been tested prospectively [3,7]. We developed an empirical model for desflurane administered in $\mathrm{O}_{2}$ with an ADU - AS $/ 5^{\circledR}$ anesthesia machine (Anesthesia Delivery Unit, General Electric, Helsinki, Finland) during the first 5 min of the anesthetic, and prospectively tested whether it allows the anesthesiologist to select a FGF - $\mathrm{F}_{\mathrm{D}}$ combination to attain a $F_{A t}$ of the inhaled anesthetic within a specified time interval (1 - $5 \mathrm{~min})$ after turning on the vaporizer.

\section{Methods}

\section{Part I. Model development}

After obtaining IRB approval (OLV Hospital, Aalst, Belgium) and written informed consent, 50 ASA physical status I or II patients presenting for plastic, urologic, or gynecologic surgery were enrolled. All patients received oral alprazolam ( 0.5 or $1.0 \mathrm{mg}) 1 \mathrm{~h}$ before the scheduled start of surgery. After preoxygenation $(8 \mathrm{~L}$. $\min ^{-1} \mathrm{O}_{2}$ FGF for $3 \mathrm{~min}$ ), propofol (3 mg. $\left.\mathrm{kg}^{-1}\right)$, rocuronium $\left(0.7 \mathrm{mg} . \mathrm{kg}^{-1}\right)$, and sufentanil $\left(0.1 \mu \mathrm{g} . \mathrm{kg}^{-1}\right)$ were administered intravenously. After tracheal intubation, ventilation was mechanically controlled by an ADU anesthesia machine. Tidal volume and respiratory rate were set fixed at $500 \mathrm{~mL}$ and 10 breaths. $\mathrm{min}^{-1}$, respectively.

In a particular patient, one (fixed) $\mathrm{O}_{2}$ FGF and desflurane $\mathrm{F}_{\mathrm{D}}$ combination was used, with the FGF ranging from 0.5 tot $5 \mathrm{~L}$. $\mathrm{min}^{-1}$ and $\mathrm{F}_{\mathrm{D}}$ from 6 to $18 \%$; this FGF - $F_{D}$ combination was chosen randomly (random function in excel). Preliminary trials indicated what combinations were likely to lead to a desflurane $\mathrm{F}_{\mathrm{A}}$ less than $1 \%$ after $2 \mathrm{~min}$ or less than $2 \%$ after $5 \mathrm{~min}$, and these were not considered.

Inspired and expired gases were analyzed by a multigas analyzer (Datex-Ohmeda Compact Airway Module M-CAiOV ${ }^{\circledR}$, Datex-Ohmeda, Helsinki, Finland) and downloaded into a spreadsheet every 10 seconds. Gases were sampled at the distal end of the endotracheal tube using a piece of sampling tubing placed through an Arndt Multi-Port Airway Adapter ${ }^{\mathbb{B}}$ (Cook Medical Inc., Bloomington, IN). Gases sampled by the gas analyzer were redirected to the anesthesia circuit via the expiratory limb. The study was terminated after 5 minutes, or earlier when the end-expired desflurane concentration had reached $8 \%$. All values above $8 \%$ were eliminated from further analysis. The $5 \mathrm{~min}$ period was somewhat arbitrarily defined as the wash-in period because it encompasses (1) anesthesia circuit wash-in; (2) FRC wash-in; (3) early uptake by the VRG; (4) and the waning effects of propofol after about $5 \mathrm{~min}$.

All measurements were done with the same anesthesia machine and gas analyzer. The $\mathrm{ADU}^{\circledR}$ circle system volume is $3.4 \mathrm{~L}$. The fresh gas flow inlet is located distal to the inspiratory valve. Fresh gas flow compensation is used to compensate for the inspiratory fresh gas flow during inspiration. The vaporizer output was measured at the common gas outlet by the same gas analyzer and compared with the dial setting using linear and non-linear regression because (1) the vaporizer output may not match the dial setting; (2) we wanted to be able to generalize the results to other $\mathrm{ADU}^{\mathbb{R}}$ units; and (3) we wanted to exclude that certain performance error patterns could be related to systematic vaporizer error. Based on 229 measurements in 48 patients in this study, the actual desflurane vaporizer output (\%) could be described as $-0.72+$ $1.075^{*}$ dial setting $\left(r^{2}=0.98\right)$; the vaporizer's output tended to increase with lower FGF (Figure 1).

A model was build to relate $\mathrm{F}_{\mathrm{A}}$ to $\mathrm{FGF}, \mathrm{F}_{\mathrm{D}}$, time, and the following patient covariates: age, height, and weight. A constant ventilation allowed us to at least standardize circuit and FRC wash-in; after $5 \mathrm{~min}$, ventilation can easily be adjusted to the desired end-expired $\mathrm{CO}_{2}$ concentration. All values before $1 \mathrm{~min}$ were deleted because zero values are hard to work with mathematically, and because the

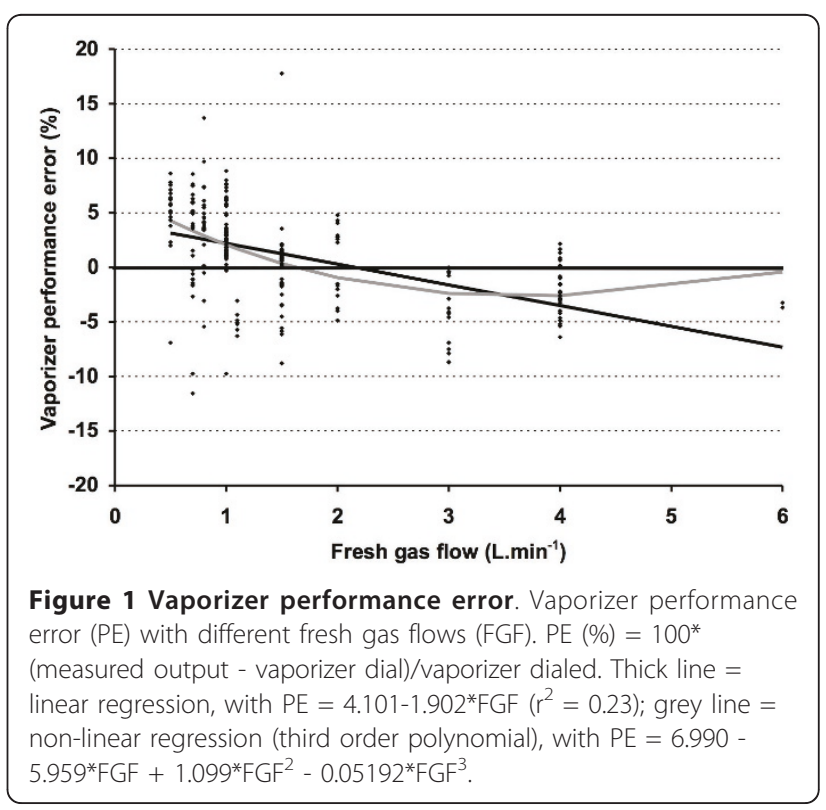


model was only supposed to model the FGF - $F_{D}$ - time relationship between 1 and $5 \mathrm{~min}$. Initial model building and parameter exploration were done in Excel using Solver ${ }^{\circledR}$ (Microsoft, Seattle, WA). The initial choice of mathematical functions was guided by three assumptions. First, because $\mathrm{F}_{\mathrm{A}}$ rises exponentially, a one exponential function was used to describe the rise of $F_{A}$. Second, the effect of a higher $F_{D}$ was modeled as curvilinear (assuming, for example, that doubling $F_{D}$ would lead to a doubling of $\left.F_{A}\right)$. Third, the effect of FGF was modeled with a single exponential (increasing as FGF is lowered). By trial and error, these and additional functions were added, deleted, modified, etc. to minimize the sum of least squares (difference between measured and predicted $\mathrm{F}_{\mathrm{A}}$ ) using Solver (Excel). Residuals were plotted against height, weight, and age to search for covariate effects by visual inspection and by linear regression. Finally, the model was tested for parsimony using NONMEM's Minimum Objective Function (ICON Development Solutions, Dublin, Ireland).

\section{Part II. Prospective testing}

The model equation derived in part I was solved for $F_{D}$ using Mathematica (Mathematica for Windows, Version 4.0, Wolfram Research Inc, Champaign, IL). The resulting equation predicts the FGF $-F_{D}$ combinations the anesthesiologist can use to attain a $F_{\text {At }}$ within the same time interval used during model development (i.e., between 1 and $5 \mathrm{~min}$ ) using a single $\mathrm{F}_{\mathrm{D}}$ and FGF setting, and takes into account the covariate effects derived during model building (see results section for actual equation).

Management of the patients during prospective testing only differed in the manner in which FGF and $F_{D}$ were selected. Patients received desflurane in $\mathrm{O}_{2}$ with the goal to reach a $\mathrm{F}_{\mathrm{At}}$ of $3.5 \%$ after $3.5 \mathrm{~min}(\mathrm{n}=40), 5 \%$ after $5 \min (n=37)$, or $6 \%$ after $4.5 \min (n=37)$. The number of patients was chosen based on prior experience. After entering the time and $\mathrm{F}_{\mathrm{At}}$ as well as significant patient covariates in the equation, the equation describes all possible FGF $-F_{D}$ combinations that reach the $\mathrm{F}_{\mathrm{At}}$ at the desired time for a patient with the particular characteristics entered into the equation. The fixed FGF that was going to be used in the individual patient was randomly selected (using Excel's random function) and entered in the equation, yielding the $\mathrm{F}_{\mathrm{D}}$ to be used. Because the resolution of the desflurane vaporizer is $0.5 \%$, the nearest value was chosen. FGF values requiring an $\mathrm{F}_{\mathrm{D}}$ above the vaporizer limit (18\%) obviously could not be tested.

To allow us to compare model performance between the three subgroups (3.5\% after $3.5 \mathrm{~min}, 5 \%$ after $5 \mathrm{~min}$, or $6 \%$ after $4.5 \mathrm{~min}$ ), the performance error (PE) for each patient was calculated as $100 *\left(\left(\mathrm{~F}_{\mathrm{A}}\right.\right.$ measured $-\mathrm{F}_{\mathrm{A}}$ predicted $) / \mathrm{F}_{\mathrm{A}}$ predicted), and the absolute performance error (APE) as the absolute value of PE. Next, for each subgroup, the following were examined: (1) bias and accuracy, using the median performance error (MDPE, median of all $\mathrm{PE}$ ) and median absolute performance error (MDAPE, median of all APE) [8]; (2) the relationship between FGF and PE (and APE) using linear regression (linear correlation) and a third order polynomial (non-linear effects) to help assess whether the model systematically over- or underestimated the end-expired desflurane concentration with increasing FGF.

\section{Results}

\section{Part I. Model derivation}

Patient demographics are presented in Table 1. The following equation described measured $\mathrm{F}_{\mathrm{A}}$ best:

$$
\begin{aligned}
& \mathrm{F}_{\mathrm{A}}=\left(1 /(\mathrm{Ht} * 0.1) *\left(2.55 * \mathrm{FGF}+1348 *\left(1-\mathrm{e}^{(-\mathrm{FGF} *-0.23)}\right.\right.\right. \\
& \left.\left.+2.49 *\left(1-\mathrm{e}^{(-\mathrm{FGF} * 0.24)}\right) * 0.39 * \mathrm{~F}_{\mathrm{D}}\right) *\left(0.03 *\left(1-\mathrm{e}^{(\text {Time }-4.08)}\right)\right)\right),
\end{aligned}
$$

with $\mathrm{Ht}=$ height $(\mathrm{cm})$, Time $=$ time after turning on the vaporizer ( $\mathrm{min}$ ), and with $\mathrm{F}_{\mathrm{A}}, \mathrm{F}_{\mathrm{D}}$ and FGF expressed in $\%, \%$, and $\mathrm{L}$. $\mathrm{min}^{-1}$, respectively. The measured versus model predicted $\mathrm{F}_{\mathrm{A}}$ are presented in Figure 2. Only height decreased the NONMEM minimum objective function significantly, and therefore is the only patient covariate to be incorporated into the model. Plots of the residuals versus patient covariates are presented in Figure 3. Note that the rebreathing function $\left(1-\mathrm{e}^{(-\mathrm{FGF}}-\right.$ $\left.{ }^{0.23)}+2.49^{*}\left(1-\mathrm{e}^{(-\mathrm{FGF} 0.24)}\right)^{*} 0.39^{*} \mathrm{~F}_{\mathrm{D}}\right)$, will cause the $\mathrm{F}_{\mathrm{A}}$ to decrease again at high flows, an effect caused by a decreased vaporizer output with high FGF due to cooling.

\section{Part II. Prospective testing}

Solving the above derived equation for $\mathrm{F}_{\mathrm{D}}$ yields the following equation:

$$
\begin{aligned}
& \mathrm{FD}=-\left(\mathrm { e } ^ { ( - \mathrm { FGF } * 0 . 2 3 + \mathrm { FGF } * 0 . 2 4 ) } * \left(\mathrm{e}^{(\mathrm{FGF} * 0.23)} * \mathrm{~F}_{\mathrm{At}} * \mathrm{Ht} * 0.1-\mathrm{e}^{(\mathrm{FGF} * 0.23)}\right.\right. \\
& * \mathrm{FGF} * 2.55+40.46-\mathrm{e}^{(\mathrm{FGF} * 0.23)} * 40.46+\mathrm{e}^{(\mathrm{FGF} *-0.23+\mathrm{Time} / 4.08)} * 40.46 \\
& \left.\left.-\mathrm{e}^{(\text {Time } / 4.08)} * 40.46\right)\right) /\left(\left(-1+\mathrm{e}^{(\mathrm{FGF} * 0.24)}\right) *\left(-1+e^{(\text {Time } /-4.08)}\right) * 39.29\right)
\end{aligned}
$$

where Time $=$ desired time lag after turning on the vaporizer to reach $\mathrm{F}_{\mathrm{At}}$ (any value between 1 and $5 \mathrm{~min}$ ); and $\mathrm{Ht}=$ patient height in $\mathrm{cm}$. After entering $\mathrm{F}_{\mathrm{At}}$, Time, $\mathrm{Ht}$, and chosen FGF, the corresponding $\mathrm{F}_{\mathrm{D}}$ to reach $\mathrm{F}_{\mathrm{At}}$ within the desired time lag can be calculated. Because any FGF can be chosen, this will result in a virtually infinite number of FGF - $F_{D}$ combinations. Patient demographics for each subgroup are presented in Table 1. Performance parameters PE, MDPE, APE and MDAPE for the three subgroups are presented in Figure 4, that also includes the linear regression line and a third order polynomial fit to examine whether PE and APE systematically increased or decreased with increasing FGF. MDPE and MDAPE were -2.9 and $7.0 \%$ in the 
Table 1 Patient demographics, presented as mean (standard deviation)

\begin{tabular}{lcccc}
\hline Group & Number & Age (years) & Weight $\mathbf{( k g )}$ & Height (cm) \\
\hline Model derivation group & 50 & $52(15)$ & $70(14)$ & $166(9)$ \\
$3.5 \%$ after 3.5 min group & 40 & $46(16)$ & $72(12)$ & $170(9)$ \\
$5 \%$ after 5 min group & 37 & $53(18)$ & $77(16)$ & $170(8)$ \\
6\% after 4.5 min group & 37 & $52(16)$ & $73(14)$ & $169(9)$ \\
\hline
\end{tabular}

$3.5 \%$ after 3.5 min group, -3.4 and $11.4 \%$ in the $5 \%$ after 5 min group, and -16.2 and $16.2 \%$ in the $6 \%$ after 4.5 min groups, respectively. There was a very weak (linear) correlation between $\mathrm{F}_{\mathrm{A}}$ and age, but not with height (effect incorporate in model) and weight (Figure 5).

\section{Discussion}

An empirical equation can be derived that predicts FGF - $F_{D}$ combinations that attain a $F_{A t}$ of an inhaled anesthetic after a predefined time lag. With modern computing power such an equation can easily be entered into an Excel file to calculate the required $F_{D}$ for any chosen FGF. While our current model will need to be refined and tested for different patient populations, agents, carrier gases, and anesthesia machines, our results prove the concept.

The concept of using an equation to predict the required $F_{D}$ for any chosen FGF to help the anesthesiologist attain and maintain a $F_{A t}$ is not new. Three decades ago, Lowe described a "general anesthetic equation" that describes the FGF - $\mathrm{F}_{\mathrm{D}}$ relationship in a circle breathing system [4]. That equation was derived by considering

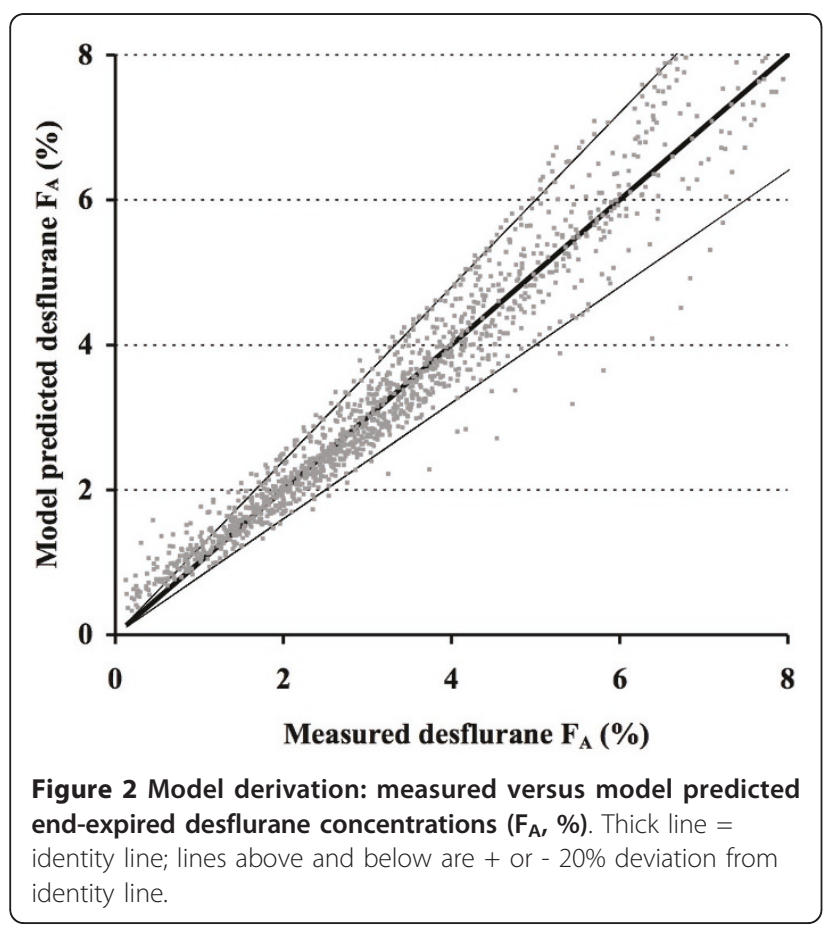

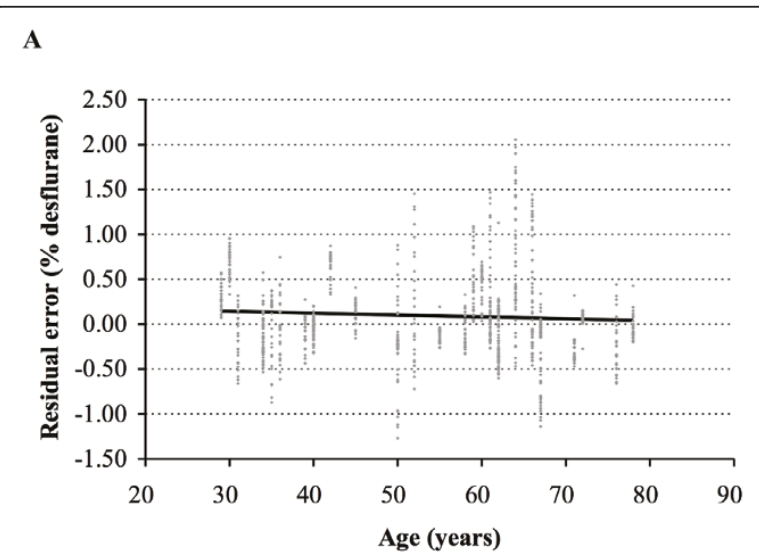

B

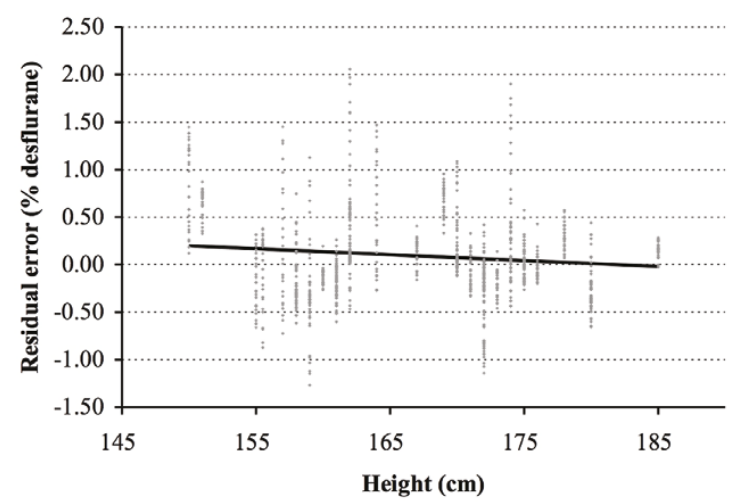

C

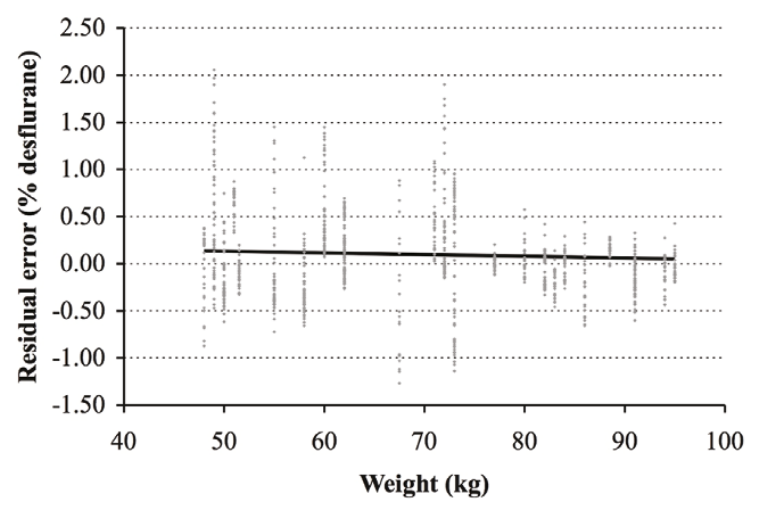

Figure 3 Model derivation: final plot of residual error (modeled minus measured $F_{A}$ ) versus patient covariates. $A=$ age, $B=$ height, and $C=$ weight. There was no (linear) correlation with age $\left(r^{2}=0.00\right)$, height $\left(r^{2}=0.01\right.$ after implementing it as a covariate in the model) and weight $\left(r^{2}=0.00\right)$. 


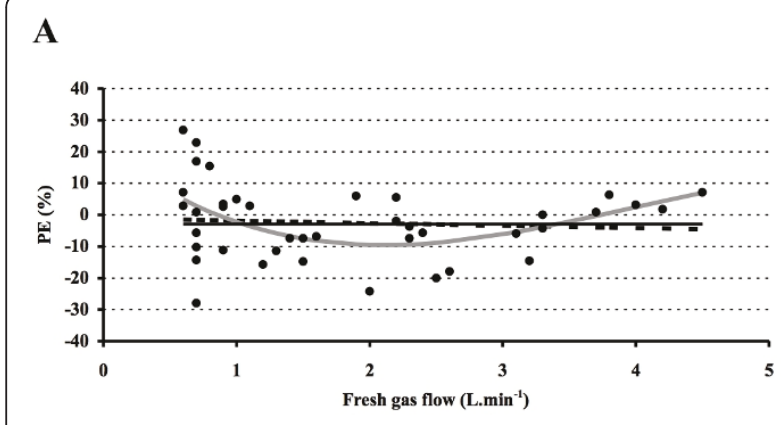

C

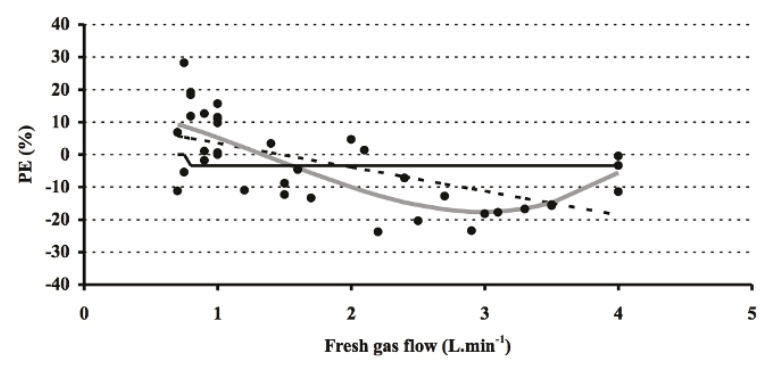

$\mathbf{E}$

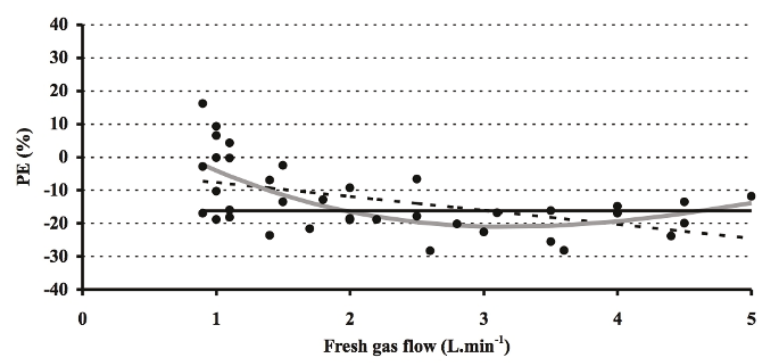

B

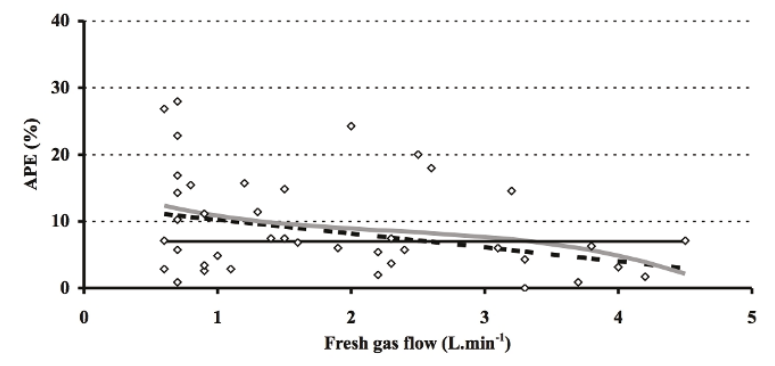

D

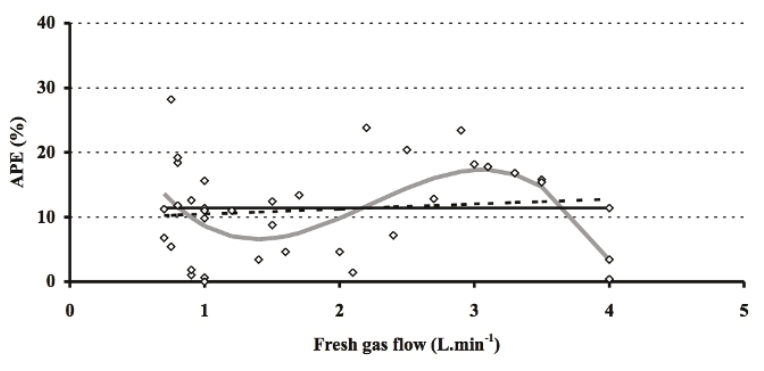

$\mathbf{F}$

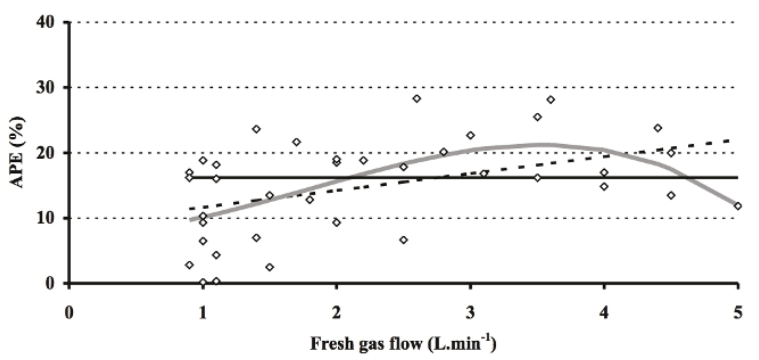

Figure 4 Model performance. Performance Error (PE, \%, closed circles, group 3.5\% after $3.5 \mathrm{~min}=\mathrm{A}$; group $5 \%$ after 5 min = C, and group 6\% after 4 min = E) and Absolute Performance Error (APE, \%, open circles, group 3.5\% after 3.5 min = B, group 5\% after 5 min = D, and group 6\% after 4 min = F), with their respective linear regression line (hatched), polynomial fit (grey line), and median performance error (MDPE, continuous black line, A, C, and E) and median absolute performance error (MDAPE, continuous black line, B, D, and F).

mass balances in the circle system and by making certain assumptions regarding the uptake pattern of the agent (the square root of time model) [4]. The model was mainly developed to facilitate the use of closed circuit anesthesia, and has never been tested prospectively across the entire FGF spectrum. Our current empirical model only describes the first 5 min of inhaled agent administration, the wash-in phase, and is to be combined (in the future) with a model that predicts the FGF - $F_{D}$ relationship during the maintenance phase.

The MDPE $(-2.9,-3.4$, and $-16.2 \%)$ as well as the MAPDE $(7.0,11.4$, and $16.2 \%)$ are within the limits deemed acceptable for target controlled infusion systems for intravenous anesthetic agents (MDPE $<10-20 \%$ and MDAPE 20 - 40\%) [9]. Still, there is some degree of misspecification of the model that could not be accounted for: there are some outliers of $30 \%$, and the error seems larger in the lower FGF range. The latter could be explained by the fact that uptake differs almost 170\% among patients $[10,11]$. This variability in uptake may have a more pronounced effect on $\mathrm{F}_{\mathrm{A}}$ with lower FGF because increased rebreathing causes the effect of the (unpredictable) amount of uptake on the composition of the inspired mixture to become more pronounced. During modeling, attempts are made to improve the degree of misspecification by taking the effect of covariates into account. However, only height significantly decreased the minimum objective function. Weight (within the range encountered in the study population) has previously been shown not to correlate with agent uptake [10-13]. Therefore, it is no surprise that weight did not improve the minimum objective function during model development, and that there was no significant effect of weight as a covariate. Nevertheless, future modeling in a still larger patient group might reveal 


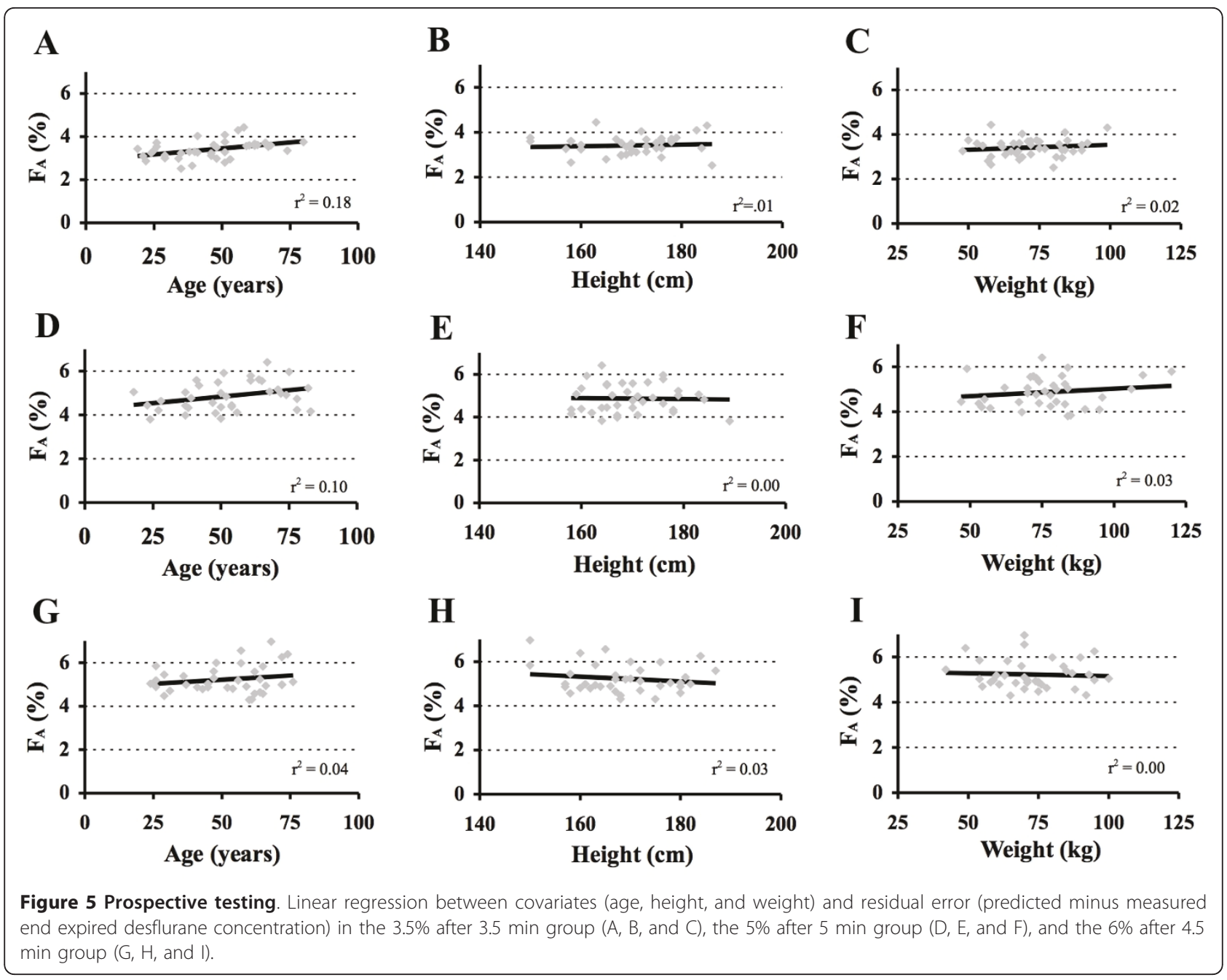

for example that lean body weight might be a useful covariate. Age did not improve NONMEMs minimum objective function during model development either, but visual inspection of the $\mathrm{F}_{\mathrm{A}}$ versus age plot during prospective testing suggests $\mathrm{F}_{\mathrm{A}}$ to be higher with older age $\left(\mathrm{r}^{2}\right.$ ranged from $0.04-0.18$ ). Age might therefore still turn out to be a useful covariate in a new model based on a larger number of patients. Other factors may be important - it may be that model misspecification increases with altered physiology and pathophysiology, e.g. when older, sicker patients are included (patients in this study were relatively healthy - ASA 1-2), when cardiac output is altered, or when synergistic effects are likely to come into play (e.g. with premedication).

Other limitations exist. The wash-in model may not be applicable when spontaneous ventilation is allowed immediately following intravenous induction of anesthesia, because the irregular and inconsistent breathing at that time does not allow the acquisition of reliable endexpired concentrations. This also is an issue for modern anesthesia machines that use automated closed-loop end-tidal feedback administration of inhaled agents. Also, the model is limited to a maximum end-expired concentration of $8 \%$ - higher concentrations might be needed in some patients, but this comes at a risk of irritating the airway.

An empirical equation that describes the FGF - $F_{D}$ relationship may have some interesting applications. It can be used to predict and depict the course of $\mathrm{F}_{\mathrm{A}}$ in the individual patient over a wide range of FGF - $F_{D}$ settings during the first $5 \mathrm{~min}$ of an anesthetic. In figure $6 \mathrm{~A}$, the equation has been solved for a $176 \mathrm{~cm}$ tall patient to describe the $F_{A}$ resulting from different FGF - $F_{D}$ combinations after 1, 2, 3, 4, and 5 min (multi-colored graphs, bottom to top, respectively; more detail on how this graph was derived can be found in the Appendix). The equation can further be used to calculate the FGF $-F_{D}$ combinations that attain a $F_{A t}$ after a predefined time interval. If the $\mathrm{F}_{\mathrm{At}}$ would be $4.5 \%$ (the light blue surface), the required FGF - $\mathrm{F}_{\mathrm{D}}$ combinations that attain $4.5 \%$ 

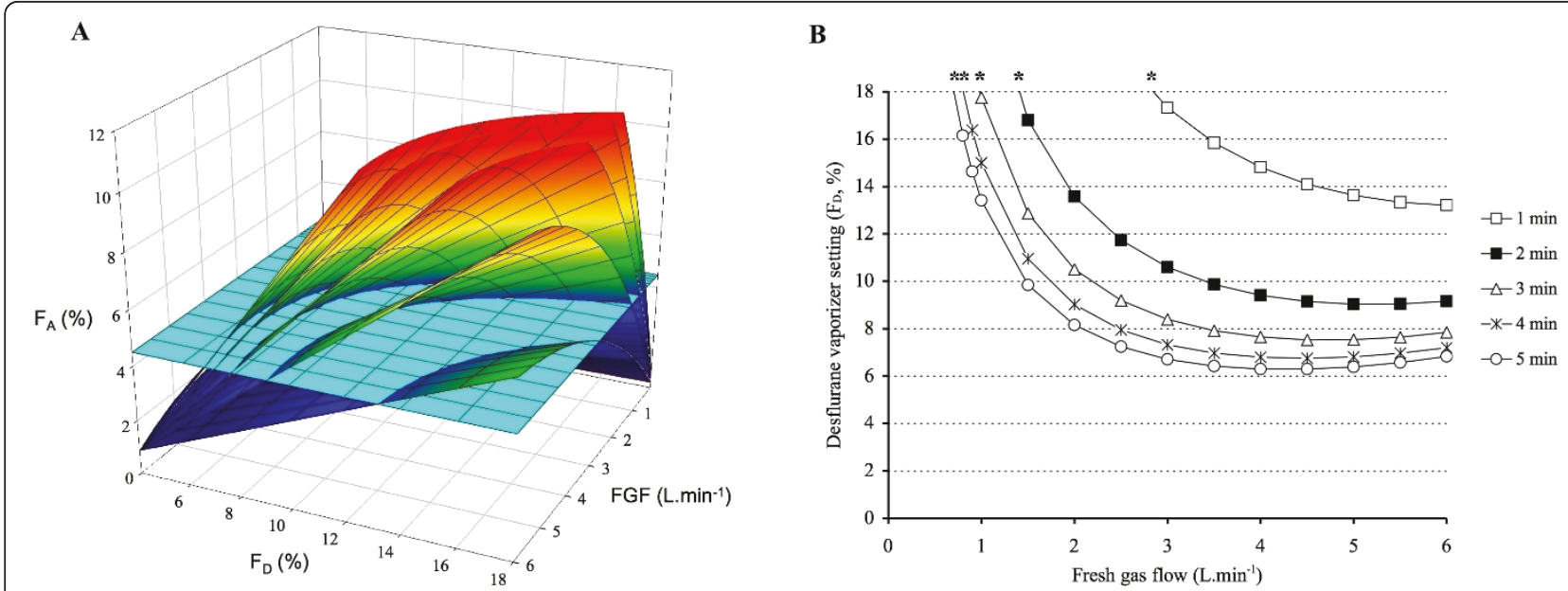

Figure 6 Visual presentation of model-based desflurane $F_{A}$ course in a fictive $176 \mathrm{~cm}$ tall patient. The multi-colored graphs (A) describe the predicted $F_{A}$ resulting from different FGF - $F_{D}$ combinations after 1, 2, 3, 4, and 5 min (bottom to top, respectively). The intersection of these surfaces with the $F_{A t}$ surface, in this example $4.5 \%$ desflurane (light blue horizontal surface), yields the corresponding FGF - $F_{D}$ combinations that attain that $\mathrm{F}_{\mathrm{At}}(\mathrm{B})$. The asterixes $\left(^{*}\right)$ indicate the lowest possible FGF that can be used to attain $4.5 \%$ desflurane at $1,2,3,4$, and 5 min because of the maximum $18 \%$ vaporizer setting. More details are provided in the Appendix.

after $1,2,3,4$, and 5 min can be found by calculating the intersection between the multicolored surfaces and the light blue surface. These intersections are presented in Figure 6B. This figure also illustrates how the equation can be used to calculate the lowest FGF that could be used with the maximum $18 \%$ vaporizer setting to attain a certain $\mathrm{F}_{\mathrm{At}}$ (i.e., it illustrates the FGF below which vaporizer output becomes inadequate to attain the $\mathrm{F}_{\mathrm{At}}$ within the specified time interval).

\section{Conclusion}

An empirical model is described that allows the derivation of a formula for each type of anesthesia machine that predicts the FGF - $F_{D}$ combinations in a circle breathing system that attain a target end-expired agent concentration in a particular patient within a specified time interval $(1-5 \mathrm{~min})$ after turning on the vaporizer. The sequences are easily calculated in an Excel file and simple to use (one fixed FGF - $\mathrm{F}_{\mathrm{D}}$ setting), and have the potential to minimize agent consumption and reduce pollution by allowing to determine the lowest possible FGF that can be used.

\section{Appendix: Derivation of Figure 6}

According to the model, $\mathrm{F}_{\mathrm{A}}=(1 /(\mathrm{Ht} * 0.1)) *\left(2.55^{*} \mathrm{FGF}\right.$ $+1348^{*}\left(1-\mathrm{e}^{\left(-\mathrm{FGF} F^{*}-0.23\right)}+2.49^{*}\left(1-\mathrm{e}^{\left(-\mathrm{FGF}{ }^{*} 0.24\right)}\right)^{*} 0.39^{*} \mathrm{~F}_{\mathrm{D}}\right)^{*}\left(0.03^{*}\right.$ $\left.\left.\left(1-\mathrm{e}^{(\text {Time/-4.08) }}\right)\right)\right)$ with $\mathrm{Ht}=$ height $(\mathrm{cm})$, Time = time after turning on the vaporizer (min), and with $\mathrm{F}_{\mathrm{A}}, \mathrm{F}_{\mathrm{D}}$ and FGF expressed in \%, \%, and L. $\min ^{-1}$, respectively.

Let us examine how the desflurane $\mathrm{F}_{\mathrm{A}}$ would evolve for a $176 \mathrm{~cm}$ tall patient over the entire $F G F-F_{D}$ range studied during model development. The surface describing desflurane $\mathrm{F}_{\mathrm{A}}$ after $3 \mathrm{~min}$ is calculated by entering the following parameters into the above formula: $\mathrm{Ht}=$ 176; Time $=3$; and a sufficient number of FGF $-F_{D}$ combinations to allow the reconstruction of a surface in Sigmaplot (Systat Software Inc, San Jose, CA, USA). The multicolored surface in Figure 7 represents these predicted $F_{\mathrm{A}}$ values after 3 min.

To determine which FGF - $\mathrm{F}_{\mathrm{D}}$ combinations result in a $F_{\text {At }}$ of e.g. $4.5 \%$ after $3 \mathrm{~min}$, the intersection has to be calculated between this target (the horizontal light blue surface) and the multicolored surface that describes the $F_{A}$ values with the entire $F G F-F_{D}$ range after $3 \mathrm{~min}$ (Figure 7). This line can be calculated by entering $\mathrm{Ht}=$ 176 , Time $=3$, and $F_{A t}=4.5$ in the formula $F_{D}=-(e$

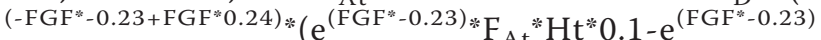
"FGF"2.55+40.46-e $\mathrm{e}^{(\mathrm{FGF}-0.23) * 40.46+\mathrm{e}^{(\mathrm{FGF}-0.23+\text { Time } /-4.08)}}$ *40.46- $\left.\left.\mathrm{e}^{(\text {Time/-4.08)* }} 40.46\right)\right) /\left(\left(-1+\mathrm{e}^{(\mathrm{FGF} 0.24)}\right)^{*}\left(-1+\mathrm{e}^{(\text {Time } /-}\right.\right.$ $4.08) * 39.29)$. This intersection line is shown in Figure 8 and presents the virtually infinite number of FGF - $F_{D}$ combinations that result in an $\mathrm{F}_{\mathrm{A}}$ of $4.5 \%$ after $3 \mathrm{~min}$ in a $176 \mathrm{~cm}$ tall patient. It also illustrates that it is not possible to achieve this target with a FGF lower than $0.7 \mathrm{~L}$. $\min ^{-1}$ because $F_{D}$ would have to be higher than the maximum $18 \%$. Note that the curve increases at high FGF because vaporizer output decreases due to excessive cooling.

Similar graphs can be developed for different time intervals (between 1 and $5 \mathrm{~min}$ ). The multicolored surface in Figure 7 describes the desflurane $\mathrm{F}_{\mathrm{A}}$ after using a range of FGF $-F_{D}$ combinations for $5 \mathrm{~min}$; the 
A

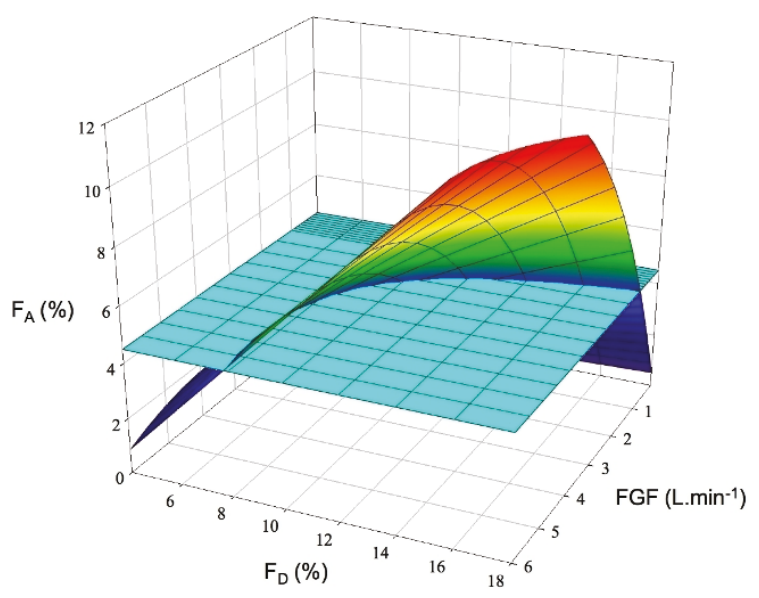

B

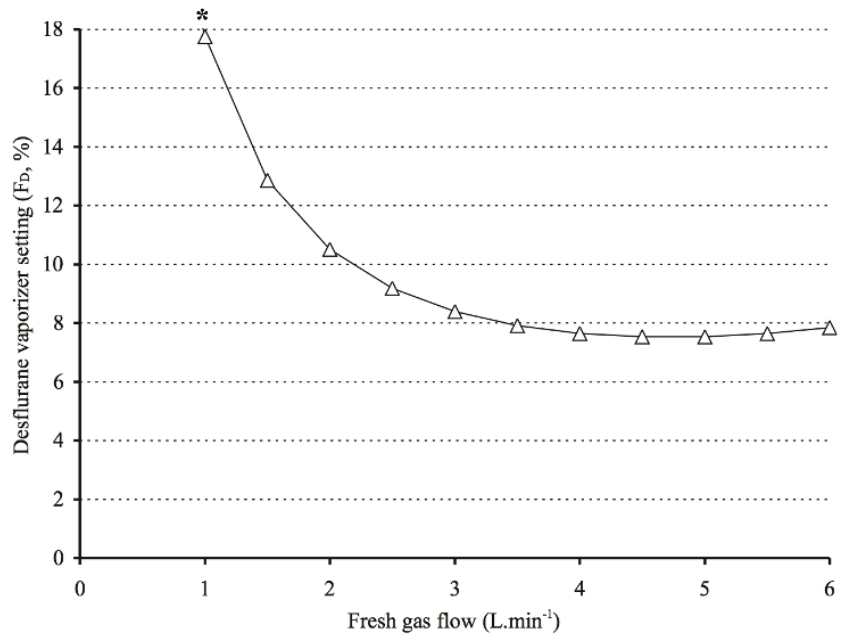

Figure 7 Multicolored surface describing desflurane $F_{A}$ after $3 \mathrm{~min}$ for a $176 \mathrm{~cm}$ tall patient over the FGF - $F_{D}$ range studied. The FGF $F_{D}$ combinations that result in a $F_{A t}$ of $4.5 \%$ (light blue horizontal surface) after $3 \mathrm{~min}$ are found by calculating the intersection between this target (the light blue surface) and the multicolored surface; these FGF - $F_{D}$ combinations are presented in $B$. The asterix (*) indicates the lowest possible FGF that can be used to attain $4.5 \%$ desflurane at 3 min because of the maximum 18\% vaporizer setting.

A

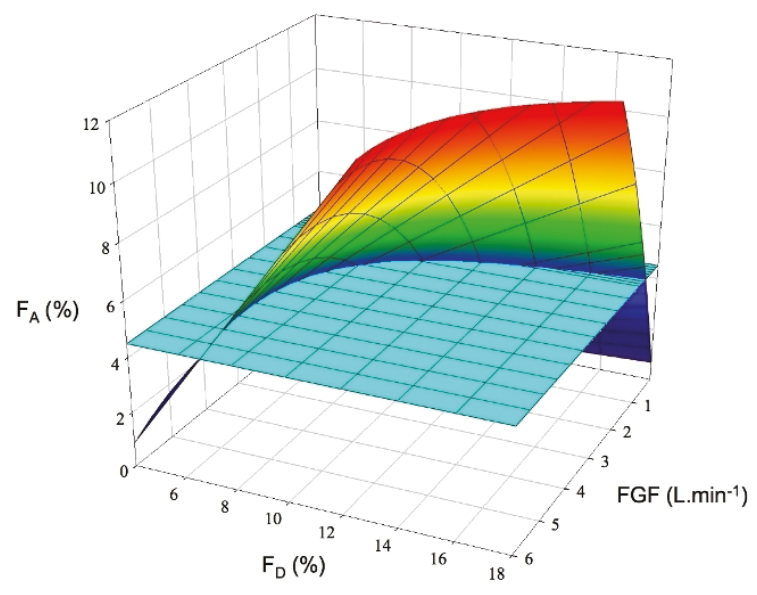

B

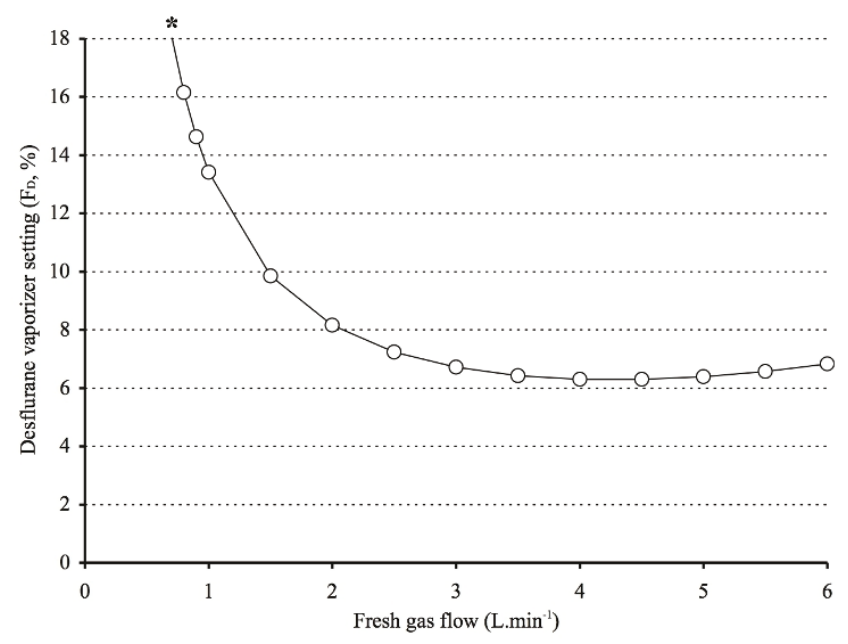

Figure 8 Multicolored surface describing desflurane $F_{A}$ after $5 \mathrm{~min}$ for a $176 \mathrm{~cm}$ tall patient over the FGF - $F_{D}$ range studied. The FGF $F_{D}$ combinations that result in a $F_{A t}$ of $4.5 \%$ (light blue surface) after 5 min are found by calculating the intersection between this target (the light blue surface) and the multicolored surface; these FGF - $F_{D}$ combinations are presented in B. The asterix (*) indicates the lowest possible FGF that can be used to attain $4.5 \%$ desflurane at 5 min because of the maximum $18 \%$ vaporizer setting.

intersection between this surface and the target $4.5 \%$ surface (horizontal light blue surface) thus describes the virtually infinite number of FGF - $\mathrm{F}_{\mathrm{D}}$ combinations that result in an $\mathrm{F}_{\mathrm{A}}$ of $4.5 \%$ after $5 \mathrm{~min}$ in a $176 \mathrm{~cm}$ tall patient (Figure 8). Figure 6 is a composite of these graphs for the time intervals of $1,2,3,4$, and $5 \mathrm{~min}$ with a $\mathrm{F}_{\mathrm{At}}$ of $4.5 \%$.

\section{Author details}

'Department of Anesthesiology, Intensive Care and Pain Therapy, Onze Lieve Vrouwziekenhuis, Aalst, Belgium. ${ }^{2}$ Department of Anesthesia, Stanford School of Medicine, Stanford, California, USA. ${ }^{3}$ Department of Anesthesiology, SintJan Hospital, Brussels, Belgium. ${ }^{4}$ Department of Anesthesiology, Intensive Care and Pain Therapy, Catharina Hospital, Eindhoven, The Netherlands. ${ }^{5}$ Department of Clinical Pharmacy, Catharina Hospital, Eindhoven, The Netherlands. ${ }^{6}$ Department of Anesthesiology, University of Ghent, Ghent, Belgium. ${ }^{7}$ Department of Anesthesiology, Northwestern University Medical School, Chicago, Illinois, USA. 


\section{Authors' contributions}

$\mathrm{JH}, \mathrm{HL}$ and ADW conceptualized the idea, designed the study, and performed mathematical analysis. All authors participated in data analysis and interpretation and drafting of the manuscript. All authors read and approved the final manuscript.

\section{Competing interests}

Jan Hendrickx has received speaker fees and reimbursement of travel expenses related to presentation of his work from GE, Abbott, and Baxter.

Received: 7 December 2010 Accepted: 24 June 2011

Published: 24 June 2011

\section{References}

1. Hendrickx JFA, Vandeput DM, De Geyndt AM, De Ridder KP, Haenen JS, Deloof T, De Wolf AM: Maintaining sevoflurane anesthesia during lowflow anesthesia using a single vaporizer setting change after overpressure induction. J Clin Anesth 2000, 12:303-7.

2. De Cooman S, De Mey N, Dewulf BB, Carette R, Deloof T, Sosnowski M, De Wolf AM, Hendrickx JF: Desflurane consumption during automated closed-circuit delivery is higher than when a conventional anesthesia machine is used with a simple vaporizer- $\mathrm{O}_{2}-\mathrm{N}_{2} \mathrm{O}$ fresh gas flow sequence. BMC Anesthesiol 2008, 8:4.

3. Lerou JG, Verheijen R, Booij LH: Model-based administration of inhalation anaesthesia. 4. Applying the system model. Br J Anaesth 2002, 88:175-83.

4. Lowe HJ, Ernst EA: The quantitative practice of anesthesia - use of closed circuit. Baltimore, Williams \& Wilkins; 1981.

5. Lerou JG, Dirksen R: A system model for closed-circuit inhalation anesthesia. I. Computer study. Anesthesiology 1991, 75:345-55.

6. Mapleson WW: The theoretical ideal fresh-gas flow sequence at the start of low-flow anaesthesia. Anaesthesia 1998, 53:264-72.

7. Ip-Yam PC, Goh MH, Chan YH, Kong CF: Clinical evaluation of the Mapleson theoretical ideal fresh gas flow sequence at the start of lowflow anaesthesia with isoflurane, sevoflurane and desflurane. Anaesthesia 2001, 56:160-4.

8. Varvel JR, Donoho DL, Shafer SL: Measuring the predictive performance of computer-controlled infusion pumps. J Pharmacokinet Biopharm 1992, 20:63-94.

9. Swinhoe CF, Peacock JE, Glen JB, Reilly CS: Evaluation of the predictive performance of a 'Diprifusor' TCl system. Anaesthesia 1998, 53:61-7.

10. Westenskow DR, Jordan WS, Hayes JK: Uptake of enflurane: a study of the variability between patients. Br J Anaesth 1983, 55:595-601.

11. Lockwood GG, Chakrabarti MK, Whitwam JG: The uptake of isoflurane during anaesthesia. Anaesthesia 1993, 48:748-52.

12. Hendrickx JF, Soetens M, Van der Donck A, Meeuwis H, Smolders F, De Wolf AM: Uptake of desflurane and isoflurane during closed-circuit anesthesia with spontaneous and controlled mechanical ventilation. Anesth Analg 1997, 84:413-8.

13. Hendrickx JFA, Van Zundert AAJ, De Wolf AM: Sevoflurane pharmacokinetics: effect of cardiac output. Br J Anaesth 1998, 81:495-501.

\section{Pre-publication history}

The pre-publication history for this paper can be accessed here: http://www.biomedcentral.com/1471-2253/11/13/prepub

doi:10.1186/1471-2253-11-13

Cite this article as: Hendrickx et al:: Mathematical method to build an empirical model for inhaled anesthetic agent wash-in. BMC

Anesthesiology 2011 11:13.

\section{Submit your next manuscript to BioMed Central and take full advantage of:}

- Convenient online submission

- Thorough peer review

- No space constraints or color figure charges

- Immediate publication on acceptance

- Inclusion in PubMed, CAS, Scopus and Google Scholar

- Research which is freely available for redistribution

Submit your manuscript at www.biomedcentral.com/submit
Biomed Central 